C.J. van Oss and R.F. Giese

\title{
Properties of two species of deadly nano-needles
}

ABSTRACT. There is a current surge of interest in the hazards associated with human exposure to nanoparticles. Nevertheless, the dangers of some naturally nanoparticulate minerals are well known. This paper reviews existing data in order to elucidate the common features conferring toxicity, which are also possessed, it is concluded, by novel manufactured nanofibres such as carbon nanotubes and gold nanorods.

Nanotechnology Perceptions 5 (2009) 147-150

Nonsubscribers: purchase individual article 